\title{
SVM significant role selection method for improving semantic text plagiarism detection
}

\author{
Ahmed Hamza Osman*, Omar M. Barukab \\ Faculty of Computing and Information Technology, King Abdulaziz University, Jeddah 21911, Saudi Arabia
}

\section{A RT ICLE INFO}

\section{Article history}

Received 21 April 2017

Received in revised form

13 July 2017

Accepted 14 July 2017

\section{Keywords:}

Plagiarism detection

Semantic similarity

Semantic role

SVM classifier

NLP

\begin{abstract}
A B S T R A C T
This research introduces an approach for the prediction and detection of plagiarized text based on Semantic Role Labelling (SRL) and Support Vector Machine (SVM). The introduced method evaluates and analyses text based on semantic position for each term within the text. It additionally detects the source semantic sense in considering the connections between its terms using the Semantic Role Labeling (SRL). SRL presents noteworthy remuneration while creating roles from a text semantically. Selecting for every role created by the SVM method keeping in mind the end goal to foresee significant roles is a noteworthy part of the proposed system. The imperative roles that will vote by the SVM strategy will be chosen in the comparability computation process. The proposed strategy assessed utilizing the PAN-PC-10 dataset. The outcomes proved that the introduced strategy enhanced the execution as far as the assessment measures contrasted and other plagiarism detection methods.
\end{abstract}

(C) 2017 The Authors. Published by IASE. This is an open access article under the CC BY-NC-ND license (http://creativecommons.org/licenses/by-nc-nd/4.0/).

\section{Introduction}

Copyright infringement practices could be vaguer than clear, more mind boggling than inconsequential cut-and-paste. All in all, there are different types of written falsification, for example, straight plagiarism, basic copyright infringement with references, complex unoriginality utilizing commentaries, counterfeiting utilizing reference however without quotes, and rephrasing as copy. The act of counterfeiting is a type of scholastic great injustice since it undercuts the whole academic initiative. Copied news, magazines, web assets and articles are the territory of worry in this similarity issue. This study led an enhancement research intended for semantic similarity recognition to expose the concealed plagiarism performs dedicated by academy scholars and to explore the researcher's involvement in discovering copyright infringement. The review guaranteed that college teachers require mechanized answers with the end goal of distinguishing thought written falsification. Summarizing is a procedure to alter the shape of a unique text by changing the structure of the sentence or replaces a portion of the first terms with its

\footnotetext{
* Corresponding Author.

Email Address: ahoahmad@kau.edu.sa (A. H. Osman) https://doi.org/10.21833/ijaas.2017.08.016

2313-626X/C 2017 The Authors. Published by IASE.

This is an open access article under the CC BY-NC-ND license

(http://creativecommons.org/licenses/by-nc-nd/4.0/)
}

equivalent word. With no legitimate reference or quotes, it likewise considered as copyright infringement. One of the NLP procedures is the Semantic Role Labeling (SRL) that was utilized as a part of many fields, for example, summarization and text reduction (Salim et al., 2010), clustering and text grouping (Ozgencil et al., 2008) and text classification (Shehata et al., 2010). In this paper, an unoriginality identification scheme utilizing the SVM algorithm for choosing essential roles in light of their closeness score is proposed. SVM is a very effective arrangement system for expectation. It assesses every one of the estimations of a potential indicator highlights utilizing the SVM. This section exhibits a prologue to the change strategy for plagiarism discovery techniques utilizing SRL and SVM. SVM utilized as a highlight choice strategy to choose an essential role. The proposed technique is helpful for choosing the imperative roles from sentences. It can likewise be utilized to incredible advantage in the proposed technique for plagiarism location strategy utilizing weight roles plot that was talked about in Osman et al. (2012a) and Paul and Jamal (2015). Here, the chose roles assessed utilizing the prescient nature of SVM. This proposed strategy was utilized to recognize copy and paste copyright infringement, rephrasing or equivalent word substitution, modifying of term structure in the text, altering the sentence from aloof voice to active voice and the other way around. The SRL was utilized to dissect the text semantically. The WordNet dictionary was 
connected to separate the ideas or equivalent terms for each term in the $t$. The fundamental contrasts between the proposed strategy in this paper and alternate procedures are as per the following. Firstly, it is an extensive plagiarism identification strategy, which concentrates on many sorts of written falsifications.

\section{Literature survey}

Currently, there is no single system or copyright infringement location framework that can be named as the "best" framework in spite of the consideration that has been given to the subject of counterfeiting. The failure to figure out which is the "best" can be credited to the absence of a controlled assessment condition. Subsequently, specialists are left to build up their own techniques and analyses, which may not be re-producible.

Without concurring to measuring parameters, it is difficult to assess the nature of some of the copyright infringement discovery frameworks. This segment, for the most part, examines a portion of the use of late proposed copyright infringement discovery procedures. These procedures can be characterized into Structural techniques (Osman et al., 2010), Cluster-Based and classification techniques (Zou et al., 2010), Semantic techniques (Osman et al., 2013), Citation-Based techniques (Gipp, 2014), Cross language techniques (FrancoSalvador et al., 2016), and Syntax-Based techniques (Osman and Salim, 2013).

As indicated by Alzahrani et al. (2012), review on copyright plagiarism detection techniques, normal counterfeiting identification strategies depends on character and term-based techniques to contrast the suspected text with the unique text. The indistinguishable string-based can be recognized either precisely or somewhat utilizing charm coordinating methodologies. Stamatatos (2009) proposed another strategy named the intrinsic copyright infringement detection base on n-gram profile. This technique evaluated style varieties utilizing n-gram profile combined with a capacity in view of disparity measures as a method for finding style changes. Stamatatos (2009) additionally presented an arrangement of tenets for figuring out which reports are free from plagiarism. Ghosh et al. (2011) proposed a govern based plagiarism discovery framework utilizing a data recovery technique.

They settled an issue normal to outward plagiarism identification frameworks by utilizing an open source data recovery framework called Nutch. There were three periods of Ghosh's framework learning, planning, competitor recovery and copyright infringement discovery. Diverse strategies were worried about the composition style, for example, Gruner and Naven (2005) and Kim et al. (2005). Inherent counterfeiting utilizing Stylometric was investigated by Stein et al. (2011) and ascribed techniques for current origin to catch the style of reports that were surveyed by Stamatatos (2009).
These reviews secured numerous systems in view of Stylometric. Suárez et al. (2010) proposed a framework in view of the LempelZiv separate, which is connected to extricate auxiliary data from texts. This technique searched for anomalies in the vector of separations among every text part (Seaward and Matwin, 2009).

Elhadi and Al-Tobi (2008) presented a copy recognition procedure for linguistic structures of the archive. This method took a gander at utilizing grammatical form (POS) labels to speak to a sentence structure as a reason for more examination and investigation. This method requested and positioned the archives utilizing POS labels.

Elhadi and Al-Tobi (2009) enhanced the system of copy recognition (Elhadi and Al-Tobi, 2008) utilizing longest common Subsequence (LCS) to compute the closeness between the reports and positioned them as indicated by the most significant separated archives. Studies, for example, Koroutchev and Cebrian (2006) compacted the sentence structure of two texts in light of a standardized Lempel-Ziv (LZ) separate technique and figure the comparability of shared topological data assumed by the compressor. The system was equipped for identifying comparative text records, regardless of the possibility that they had distinctive literals. This technique alongside different strategies, for example, dealing with text reduction utilized tokenization and stops words expulsion, and was just keen on a smaller arrangement of linguistic labels.

As of late, Burrows et al. (2013) proposed another strategy to summarize procurement by means of crowd-sourcing and data mining. The suggested strategy was studied by some of the critical breaches inquired about in the copyright plagiarism capturing field, was concentrated on two issues; securing by means of crowd-sourcing, and procurement of entry level specimens. The first issue test is programmed superiority confirmation; without such a method the crowd-sourcing worldview is not powerful, and without crowdsourcing, the formation of test data is inadmissibly costly for a practical request of sizes.

Based on the discussion of related works in this section, the plagiarism detection methods still need to be improved spatially in the semantic structure category. Furthermore, in particular, utilizing the SVM-SRL scheme brought about enhanced similitude scores, not at all like any of the already proposed techniques. Whatever remains of the article is sorted out as takes after: Section 2 gives a depiction of the plagiarism detection literature review. In Section 3, a deep depiction of the fundamental thought required in the suggested strategy is secured. Section 4 examines the SVM algorithm. Corpus and data set, including execution measures, are displayed in Section 5. Section 6 examines the exploratory outline of the proposed technique. Segment 7 gives a portrayal of the outcomes and exchange of the introduced technique, while Section 8 concludes the study. 


\section{Proposed method (SRL-SVM)}

The fundamental thought of this paper is to propose a semantic plagiarism discovery strategy in light of SRL and SVM system. The proposed technique has four primary strides; which are: text preprocessing including text chunking, stemming process and stops terms expulsion; SRL; synonymy and concept exploitation and SVM determination strategy. The general framework of the proposed technique is shown in Fig. 1.

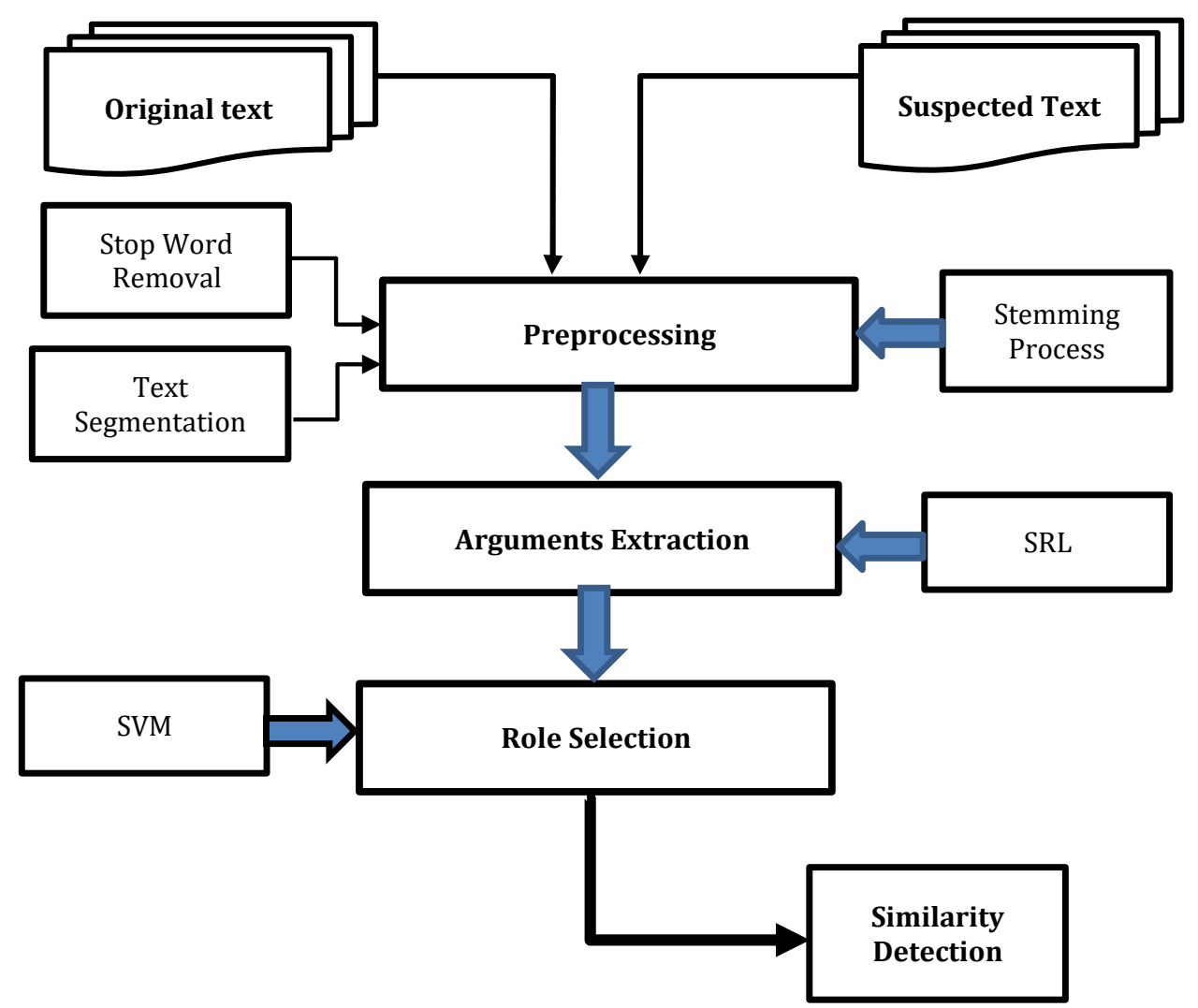

Fig. 1: Proposed method (SRL-SVM)

\subsection{Text preprocessing}

In this phase, the text preprocessing stage contained three sub-stages which were text chunk, stop words withdrawal and term stemming. A text chunk partitioned a text archive into sub-sentences. Several studies concentrates on text preparing strategies in various fields, incorporate intrusion detection (Sharma et al., 2007).

The step of stop terms removal for erasing meaningless terms was utilized. Stemming procedure to delete the attached (suffixes and prefixes) in a term to create its root term was additionally connected. This progression separated the critical terms from the text and disregarded the rest of the terms. This may have unfavorably influenced the comparability between texts.

\subsubsection{Text chunking}

Prepreparing is one of the main strides in NLP. A basic sort of prepreparing includes isolating the text into important parts and is defined text chunking. Text can be separated into words, themes, or sentences. This progression is a critical undertaking in text processing methodologies, for example, data extraction, text synopsis, semantic part naming, syntactic parsing, machine interpretation and unoriginality location.

Text chunking is conducted by limit recognition and isolating a text into sub-sentences. By and large, an outcry stamp (!), a question mark (?), or a period (.) is the typical signs that show sentences limit (Mikheev, 2000). This study utilized the sentence based text chunking as the initial phase in the suggested approach, where the first and suspected documents will be isolated into sentence pieces. This technique was picked on the grounds that the proposed strategy intends to contrast a speculated text and unique text in light of the sentence matching methodology.

\subsubsection{Stop terms removal and term stemming stage}

Stop Terms are the Terms that every now and again happen in archives. They are Terms, for example, "a", "and" and "the". These terms don't provide any indication qualities or implications to the substance of the records, henceforth; they are dispensed with from the arrangement of file words (van Rijsbergen, 1979). Tomasic and Garcia-Molina (1993) announced that such terms include about $40 \%$ to half of an accumulation of texts document terms. Disposing of the stop terms in programmer 
ordering accelerates the system comparison process, spares a gigantic amount of space in the list, and does not harm the recovery adequacy (Frakes and Baeza-Yates, 1992). Currently, different methodologies that are utilized for the assurance of such stop Terms. As of now, there are a few English stop terms records that are normally utilized as a part of data recovery. The proposed strategy dispensed with all the stop terms in the documents to accelerate the system procedure. The introduced strategy utilized the list of the Buckley stop terms (Buckley et al., 1995) that was utilized as a part of the SMART data recovery framework at Cornell University.

Terms stemming is another text preprocessing step. Currently, there are numerous English stemming tools accessible such as, Porter Stemmer, Nice Stemmer, and Text Stemmer ordinarily utilized in the NLP felid. The suggested method uses the Porter Stemmer technique to derivationally correlated types of a term to a general base frame and reduces inflectional structures. For example:

Am, is, are become (be)

books, book's, books', booking $\Rightarrow$ book

Stemming process is an answer to some of the issues required in data recovery, for example, varieties in term forms (Lennon et al., 1981). The widely recognized sorts of variety are typo mistakes, multi-term developments, substitute spellings, affixes, contractions, and translation.

\subsection{Semantic-role labelling (SRL)}

By and large, SRL is a procedure employed to recognize and name terms roles in a document (Màrquez et al., 2008). The guideline thought is that a record semantic level examination decides every one of the roles among different ideas in the archive. This can be reached out to the portrayal of levels of discourse such to decide "Verb," Object, "Subject," or "Intensifier." Through the parts naming procedure, each term in the source and suspected text is marked with their comparing parts. In this study, semanticpart marking in view of the sentence-based was suggested as a new technique for plagiarism identification. SRL intends to identify the game plan likeness among the ideas of the reports and conceivable semantic closeness among both records. This progression in the review utilized the part marks of the ideas for the text-documents and gathered them as clusters. The clusters that were utilized as a part of this technique gave a snappy manual for capturing the associated part with the text. A circumstance for the plagiarism can be shown through the accompanying illustration:

Example (1): The source text: My manager settled on the choice yesterday.

The suspected text: The choice was made by my manager yesterday.
By utilizing the SRL process, the created contentions are:

My manager made the choice yesterday

The choice was made by my manager yesterday

Figs. 2 and 3 outline the examination for suspecting sentence utilizing SRL in the mentioned case. Actually, the sentences construction of the examples above may vary if the passive versus active voice or equivalent words and synonyms are utilized. Truly, these sentences can be semantically the similar.

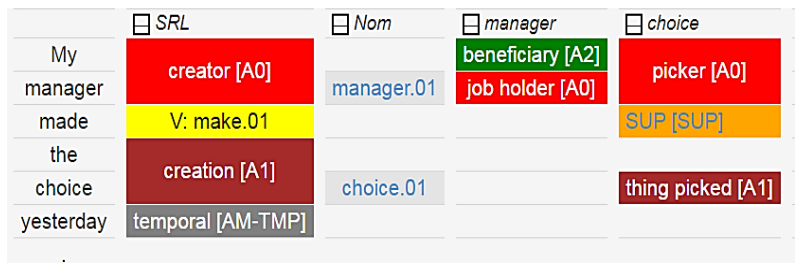

Fig. 2: SRL extraction of example 1 (source sentence)

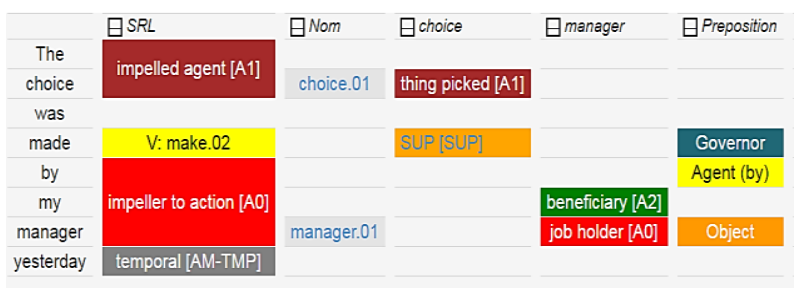

Fig. 3: SRL extraction of example 1 (suspected sentence) (Tool website: http://homepages.inf.ed.ac.uk/mroth/ demo.html)

It was noticed that the SRL highlights the role (verb, subject, adverb, and object) for a text regardless of modifying the spots for the names in the text. This highlighting supports the introduced strategy in plagiarism detection if the examination is connected in view of the roles of the sentence utilizing SRL. In the SRL similarity system (Osman et al., 2012a; Paul and Jamal, 2015), the words in the source text and the suspected text were matched. When two words discovered as similar, straightforwardly look for the role name that contains those terms and afterward think about the text that pass in these terms. This progression looks at the role names of conceivable sentences that have been plagiarized with comparing role names in unique sentences. The similarity calculation between the words must be process in the correct procedure. In the event that the proposed method think about the words in Arg0 (subject) in the suspected document with the various roles in the source document to decide the copy proportion, it cannot be right. For example, it is not reasonable to contrast the Time (Arg-TMP) and Object (Arg1) with the Verb role $(\mathrm{V})$.

In illustration 1 , the examination utilized as a part of the numerous systems, for example, string coordinating (Stein et al., 2011) or n-gram (Palkovskii et al., 2011) compares each term in the speculated text with each term in the source text. The term "manager" will be contrasted and the 
expression "choice", "settled", "manager" and "yesterday". Not only is this comparison inappropriate, as well as sets aside time for comparison. The purpose of the introduced technique is to concentrate on the comparison of the terms roles of the source text with identical terms roles in the suspected text. The introduced SRL technique can compare verb with a verb, object with an object, etc. by using this process the time of comparisons will be reduced. Every role in source text might be contrasted and a compared only with a role in suspected text.

Example (2): Assume the following text:

(0) -Original text: The fast black dog kill the sluggish fox.

(S) -Suspected text: The rapid black puppy slay the lazy canine.

To start with, sentence 0 and $\mathrm{S}$ were denoted by the cluster of terms. In cluster 0 the arrangement of terms after stemming and stop term elimination are \{fast, black, dog, kill, sluggish, fox\}, though the arrangement of terms in group B is rapid, black, puppy, slay, lazy, canine\}.

In light of the Example (2), the words of two texts $(O$ and $S)$ varied if the active versus equivalent words and synonyms are utilized. Really, these texts can be logically the similar. It was additionally noticed that the suggested strategy can detect the meaning of a text, in spite of modifying the equivalent words inside the text. This detecting assists the suggested strategy for plagiarism detection if the comparison is employed using the WordNet synonyms exploitation. Synonyms extraction is the fundamental stride in proposed recognition strategy. In this research, this is measured as determining the words with their equivalent terms from Wordnet dataset and called concept extraction step. Synonymy is one of the verbal semantic relatives, which are the correlation between the semantic of the words. In this progression, concept extraction process was conducted using thesaurus WordNet dataset.

\subsection{Support vector machine}

The Support vector machine (SVM) is a generally new technique that has immediately picked up popularity on account of the suitable outcomes that have been accomplished in a wide assortment of machine learning issues, and in light of the fact that they have strong hypothetical underpinnings in measurable learning hypothesis (Salcedo-Campos et al., 2012). SVM is a parallel classification procedure in light of factual learning hypothesis that was connected with awesome achievement in many testing nonlinear classification issues and on substantial datasets (Noble, 2006). This can be utilized to comprehend directly divisible (LS) and also non-straight distinct issues (NLS) (Temitayo et al., 2012). SVM has great speculation abilities and meets viably towards the ideal solution (Palmieri et al., 2014). Additionally, SVM is a directed learning strategy that produces input-output mapping capacities from an arrangement of marked preparing data (Wang, 2005). Prior to the disclosure of SVM, machine learning was not extremely fruitful in learning and speculation errands, with numerous issues being difficult to solve (Youn and McLeod, 2007). There are numerous kernel-based capacities, for example, straight part work, polynomial portion work, spiral premise work (RBF) and Hyperbolic Tangent (Sigmoid). Portion sigmoid capacity can be executed in SVM (Chhabra et al., 2010). For classification, nonlinear piece capacities are frequently used to change include information to a high-dimensional component space in which the info information turns out to be more distinct contrasted with the first information space. Greatest edge hyperplane was then made. SVM calculations partition the n-dimensional space portrayal of the information into two districts utilizing a hyperplane (Youn and McLeod, 2007). The created demonstrate depends just on a subset of the preparation information close to the class limits. SVM has many preferences, for example, getting the best outcome when managing parallel portrayal, ready to manage extensive quantities of components, utilizing factual learning technique, prompting to great execution without the need to fuse earlier data. The technique is exceptionally powerful in content classification field on the grounds that it can deal with highdimensional information utilizing bits to anticipate the imperative components. It can likewise utilize substantial info information and a list of capabilities, is anything but difficult to test the influence of the quantity of element on classification exactness, is stronger to the distinctive dataset and pre-preparing strategy, and a great deal more productive in preparing and managing (Jin and Ming, 2011). SVM has a few detriments, for example, it requires longer learning time, time and memory utilization when the extent of information is gigantic and preparing time can be huge if there are countless illustrations (Temitayo et al., 2012).

In this paper, SVM utilized as highlight forecast and choice technique to foresee and select the most vital contentions that were produced utilizing SRL. SVM classifiers use the hyperplane in particular classes. Each hyperplane is portrayed by its heading $(w),(b)$ is the correct position in space or a limit, (xi) is the information vector of measurement $\mathrm{N}$ or content substance and demonstrates the class. Conditions 1 and 2 show an arrangement of the preparation tests (Eq. 1).

$(\mathrm{x} 1, \mathrm{y} 1),(\mathrm{x} 2, \mathrm{y} 2), \ldots,(\mathrm{xk}, \mathrm{yk}) ; \mathrm{Xi} \in R^{d}$

where $\mathrm{k}$ is the training dataset number and $\mathrm{d}$ represents the number of dimensions of input dataset: yi $\in\{-1,+1\} ; \quad i=1,2, \ldots, k$. The decision functions of the form Eq. 2 .

$f(\mathrm{x}, \mathrm{w}, \mathrm{b})=\operatorname{sgn}((\mathrm{w} \cdot \mathrm{xi})+\mathrm{b}), \mathrm{w} \in R^{d}, \mathrm{~b} \in \mathrm{R}$ 
At that point the locale between the hyperplane, which isolates two classes, is known as the edges, which show the classification of SRL roles utilizing SVM. Give the separation from the shut data a chance to indicate the hyperplane be $\frac{1}{\|w\|}$.Among isolated hyperplane, there exists one ideal isolating hyperplane, and the separation of two support vector focuses from various sides of this hyperplane is maximal. At that point, the opposite separation from the source to this hyperplane is $\frac{1}{\|w\|}$, or the edge remove isolating hyperplane is is $\frac{2}{\|w\|}$. The base separation of the edge is equivalent to $\frac{1}{2}\|w\|^{2}$ (called primal issue) and getting the most extreme conceivable edge is the basic thought of SVM algorithm. Fig. 4 outlines the classification of bosom malignancy utilizing SVM.

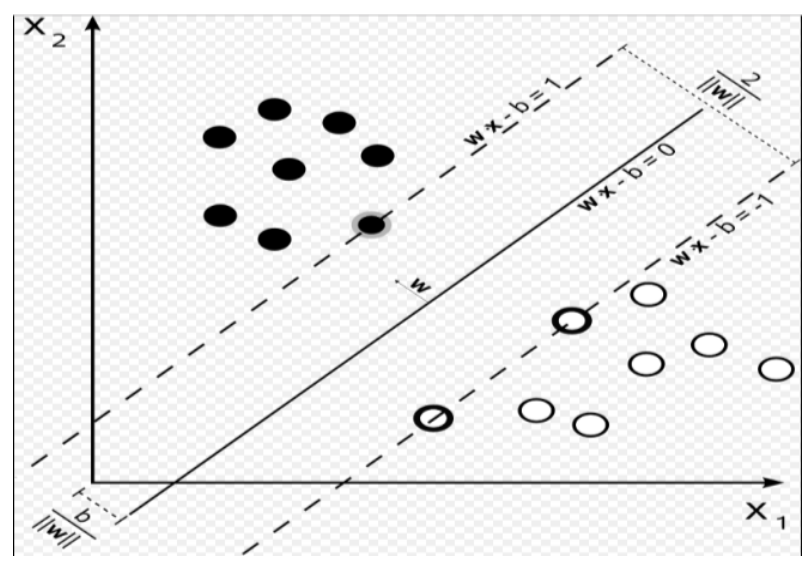

Fig. 4: Classification of plagiarism data using SVM

The proposed strategy utilized an SVM algorithm for two purposes. To start with, to choose critical predicated arguments or roles fields from each suspected and source records. At that point, test the chose roles with all arguments that were created by SRL to analyze the factual hugeness test between them. By this way, the outcomes were moved forward. Furthermore, the SVM technique was additionally utilized as an anticipated technique whereby the roles chose by the SVM turn out to be more noteworthy, particularly when it contrasted and roles that were chosen by SRL. The proposed method was connected by utilizing SRL and SVM algorithm through principle steps. The initial step was to pre-process suspected reports and unique records utilizing text division, stop words evacuation and stemming. At that point, SRL was utilized to change the text into roles in light of section for each word in the text.

The verbs of the sentences assume an essential part all the while and the comparison of the sentences. Dependence on verbs of the sentences was discussed in the related works section. Every one of the roles separated from the text was assembled by the roles sort. Every set contained also extricated roles. Each set was alluded to by their role names, for example, Arg0, Arg1, V, Time, Location, and so forth. His role's closeness score was computed in light of the SRL similitude measure proposed and portrayed by Osman et al. (2012a) and Osman et al. (2012b). Copyright infringers tend to concentrate on the imperative terms and alter them in their work. Accordingly, just imperative roles with a more prominent effect on a sentence will be focused by the literary thief. While trying to outflank the copyright infringer, a few target determination strategies are accessible, every one of them proposing to foresee the imperative focuses on the data as could be expected under the circumstances. One of these techniques is the SVM. The SVM algorithm can join measurably homogeneous qualities (roles similarity values) with the objective variable (aggregate similitude score between the roles). Utilizing this progression permits us to produce critical roles from all roles. The last stride was a likeness computation in view of the imperative roles that were created by the SVM algorithm.

\section{Plagiarism detection corpus}

The PAN-PC corpus is a multi-dialect, expansive scale, open corpus of unoriginality, containing just artificial plagiarism occurrences. Irregular appropriating tries to emulate the initiatives a human would make to shroud duplicating, muddling through the reordering of the expressions, word substitution, equivalent word, and antonym utilize, erasures, and additions. Additionally, a portion of the occasions above may likewise include interpretations of copy's section, made via programmed implies. The PAN-PC-10 corpus contains 27,073 text records, 15,925 arrangements of suspicious reports and 11,148 arrangements of source archives produced utilizing artificial plagiarism program.

The reported length shifts from one page to a few hundred pages. Half of the suspicious, reports are non-plagiarized and half contains plagiarism cases. These cases were included arbitrarily from the suspicious reports (Potthast et al., 2010a). Record and factual conveyances in the corpus are depicted in Table 1.

Table 1: Document statistics in the PAN-PC-10

\begin{tabular}{|c|c|c|c|}
\hline \multicolumn{2}{|c|}{ Document Purpose } & \multicolumn{2}{|c|}{ Plagiarism per-Document } \\
\hline Original documents & $50 \%$ & Hardly-(5\%-20\%) & $45 \%$ \\
\hline Suspected & & $-50 \%)$ & $15 \%$ \\
\hline - With- & $25 \%$ & Muc & $25 \%$ \\
\hline - Without plagiarism & $25 \%$ & Entirely-(>80\%) & $15 \%$ \\
\hline \multicolumn{2}{|l|}{ Detection Task } & \multicolumn{2}{|c|}{ Document Length } \\
\hline External-de & $70 \%$ & Short-(1-10 pp.) & $50 \%$ \\
\hline Intrinsic- & $30 \%$ & $\begin{array}{l}\text { Medium-(10-100 pp. } \\
\text { Long-(100-1000 pp.) }\end{array}$ & $\begin{array}{l}35 \% \\
15 \%\end{array}$ \\
\hline
\end{tabular}

A shortcoming of the PAN-PC is that most of the counterfeiting cases were created misleadingly.

\subsection{Performance measures}

This segment talks about execution measures of plagiarism location algorithms. The regular execution measures utilized as a part of plagiarism identification algorithms are Precision and Recall. A 
current review by Potthast et al. (2010b) introduced a smaller scale found the middle value of and a large scale arrived at the midpoint of variation. An Fmeasure or granularity is another critical measure that was utilized as a part of counterfeiting recognition evaluation. For assessing the proposed identification system, the proposed method utilized the miniaturized scale found the middle value of Precision and Recall. The smaller scale arrived at the midpoint of Precision and Recall of $\mathrm{R}$ under $\mathrm{S}$ is characterized as takes after (Eq. 3):

$\operatorname{Precision}_{\text {micro }}(S, R)=\frac{\left|U_{(s, r) \in(S \times R)}(S \sqcap R)\right|}{\left|U_{r \in R} r\right|}$

where, $\mathrm{S}$ and $\mathrm{R}$ denote sets of plagiarism cases and detections, $\mathrm{s}$ denote plagiarized passage in a plagiarized document, $r$ denote associates an allegedly plagiarized passage in a document (Eqs. 4 and 5).

$\operatorname{Recall}_{\text {micro }}(S, R)=\frac{\left|U_{(s, r) \in(S \times R)}(S \sqcap R)\right|}{\left|U_{s \in S} S\right|}$,

$S \sqcap R= \begin{cases}s \cap r & \text { if } r \text { detect } s \\ \varnothing & \text { Otherwise }\end{cases}$

where; The F-measure is the harmonic mean of precision and recall and calculated using Eq. 6 below:

$F-$ measure $=\frac{2 \times \text { Recall } \times \text { Precision }}{\text { Recall } \times \text { Precision }}$

\section{Experimental design}

The investigations inspected the measure of identifying copied sentences from the source documents. The analyses were conducted on PANPC-10 dataset (huge, medium and little size). Every one of these text was copied from at least one unique record as indicated by the PAN-PC-10 dataset. The introduced procedure was connected via looking for the presumed documents inside the clusters. The documents were equally split it and isolated into five clusters due to the huge of the dataset. Each cluster having a specific number of documents. The documents expanded for each cluster with each testing of correlation. The aim of this group procedure is to concentrate the practices of the plagiarized client for every role so it can be processed. Each group was picked as an info variable in SVM and all roles as occasions or features. Then, the yield is an aggregate comparability score over these groups. The estimations of the data variable are a closeness score between any comparable combine roles. The simility between the roles of the suspected document and source document was figured by Jaccard coefficient that can be characterized by the accompanying condition (Eq. 7):

Simialrity $\left(c_{i}\left(\operatorname{RoleS}_{\mathrm{i}}, \operatorname{RoleS}_{\mathrm{j}}\right)\right)=\frac{\mathrm{C}\left(\operatorname{RoleS}_{\mathrm{j}}\right) \cap \mathrm{C}\left(\operatorname{RoleS}_{\mathrm{k}}\right)}{\mathrm{C}\left(\operatorname{RoleS}_{\mathrm{j}}\right) \cup \mathrm{C}\left(\operatorname{RoleS}_{\mathrm{k}}\right)}$ where, $\mathrm{C}($ RoleSj $)=$ ideas of the roles sentence in the presumed report; Ci (RoleSk) = ideas of the roles sentence in the first archive; then ascertained the closeness between the suspected record and source document in light of the accompanying condition (Eq. 8):

Total simialrity ( suspected 1 , source 2$)=$

$\sum_{\mathrm{i}=1,1} \sum_{\mathrm{j}=1, \mathrm{~m}} \operatorname{SimC}_{\mathrm{i}}\left(\right.$ RoleS $\left._{\mathrm{j}}\right) \cap \mathrm{C}\left(\right.$ RoleS $\left._{\mathrm{k}}\right)$

where, SimCi (RoleSj, RoleSk) is the closeness between roles sentence $\mathrm{j}$ in speculating report containing idea $\mathrm{i}$ and roles sentence $\mathrm{k}$ in unique archive containing idea $\mathrm{i}, \mathrm{l}=$ no. of ideas, $\mathrm{m}=$ no. of Roles sentence in presumed record, $\mathrm{n}=$ no. of Roles sentence in the first record.

\section{Results and discussion}

The presumed reports were plagiarized in various methods for copyright infringement, for example, a basic copy and paste, changing a few terms with their relating equivalent words, and altering the structure of the sentences (rephrasing).

Table 2 represents the Similarity outcomes acquired from the trains performed on the chose set of documents. Each line speaks to a group of documents that are utilized to clarify the roles amid the closeness estimation.

Table 2: Similarity cross the set of clusters

\begin{tabular}{|c|c|c|c|}
\hline SRL-Type & Explanation & SRL-Type & Explanation \\
\hline Arg0 & (Agent) & NEG & $\begin{array}{l}\text { (Negation- } \\
\text { marker) }\end{array}$ \\
\hline Arg1 & $\begin{array}{l}\text { (Theme / Direct } \\
\text { object /Patient) }\end{array}$ & LOC & (Location) \\
\hline Arg2-5 & (Not-fixed) & PNC & (Purpose) \\
\hline V & (Verb) & MOD & $\begin{array}{l}\text { (Modal- } \\
\text { verb) }\end{array}$ \\
\hline MNR & (Manner) & $\mathbf{0}$ & (Adjective) \\
\hline TMP & (Time) & DIR & (Direction) \\
\hline DIS & $\begin{array}{c}\text { (Discourse- } \\
\text { connectives) }\end{array}$ & EXT & (Extent) \\
\hline ADV & (General purpose) & & \\
\hline
\end{tabular}

As demonstrated in the segments in Table 2, there are 19 roles that have been extricated utilizing the SRL. Table 3 outlines these sorts that showed up in Table 2.

Table 3: Roles sorts and their portrayals

\begin{tabular}{cccc}
\hline & Recall & Precision & F-Measure \\
\hline Cluster1 & 0.834 & 0.741 & 0.784754 \\
Cluster2 & 0.841 & 0.63 & 0.720367 \\
Cluster3 & 0.817 & 0.687 & 0.746382 \\
Cluster4 & 0.809 & 0.652 & 0.722064 \\
Cluster5 & 0.826 & 0.663 & 0.735578 \\
\hline
\end{tabular}

Table 3 demonstrates the sorts of roles that were utilized as a part of the analyses and their depiction or significance. The aftereffects of the similarity result in term of precision, recall, and f-measure are given in Table 4 . 
Table 4: Results after similarity algorithm

\begin{tabular}{ccccccccccccccccccccc}
\hline Cluster No & A0 & A0A1 & A1 & A1A0 & A2 & V & MIR & TEP & DIS & ADV & NEG & LOC & PNC & MOD & 0 & A3 & A4 & DIR & EXIT \\
\hline Cluster1 & 0.94 & 0.78 & 0.79 & 0.50 & 0.81 & 0.78 & 0 & 0 & 0 & 0.82 & 0 & 0 & 0 & 0 & 0 & 0 & 0 & 0 & 0 \\
Cluster2 & 0.83 & 0.64 & 0.93 & 0.51 & 0.92 & 0.85 & 0 & 0.80 & 0 & 0.88 & 0 & 0.85 & 0 & 0 & 0.92 & 0.80 & 0 & 0 & 0 \\
Cluster3 & 0.88 & 0.68 & 0.69 & 0.61 & 0.75 & 0.78 & 0.62 & 0.82 & 0.68 & 0 & 0 & 0.68 & 0.92 & 0 & 0.88 & 0.79 & 0 & 0 & 0 \\
Cluster4 & 0.96 & 0.79 & 0.99 & 0.86 & 0.83 & 0.78 & 0.80 & 0.76 & 0.80 & 0 & 0.78 & 0 & 0 & 0 & 0.89 & 0 & 0 & 0 & 0 \\
Cluster3 & 0.93 & 0.85 & 0.84 & 0.88 & 0.75 & 0.89 & 0 & 0.83 & 0 & 0.67 & 0 & 0.62 & 0 & 0 & 0.78 & 0 & 0 & 0 & 0 \\
\hline
\end{tabular}

Table 4 demonstrates the comparability between the source and suspected documents for each role of text. It can be watched that all the result values in recall measure are over 0.80 while all the result and incentive in precision and f-measure are more than 0.63 . Every one of the scores in Table 2 show to give great outcomes since they are more noteworthy than 0.5 yet at the same time initiatives were made to enhance these scores to get higher similarity results. After the elements forecast process using the SVM, it was noticed that the plagiarizing client does not concentrate on all roles of the sentences, thus a few roles are overlooked. These roles are called irrelevant roles. Imperative roles were chosen to enhance the similarity result by SVM process. For SVM expectation demonstrates development, the data mining tool of IBM SPSS Modular (Mikut and Reischl, 2011) has been utilized. IBM SPSS Modular was utilized as data digging programming for the proposed technique with SVM algorithm. The distinction between IBM SPSS Modular tool and different apparatuses is that its data processing is using hubs, which are then connected together to shape a stream outline. In addition, data representation and results can present to clients in the wake of mining procedure has been finished. Fig. 5 shows the selected roles of the introduced strategy using the SVM technique.

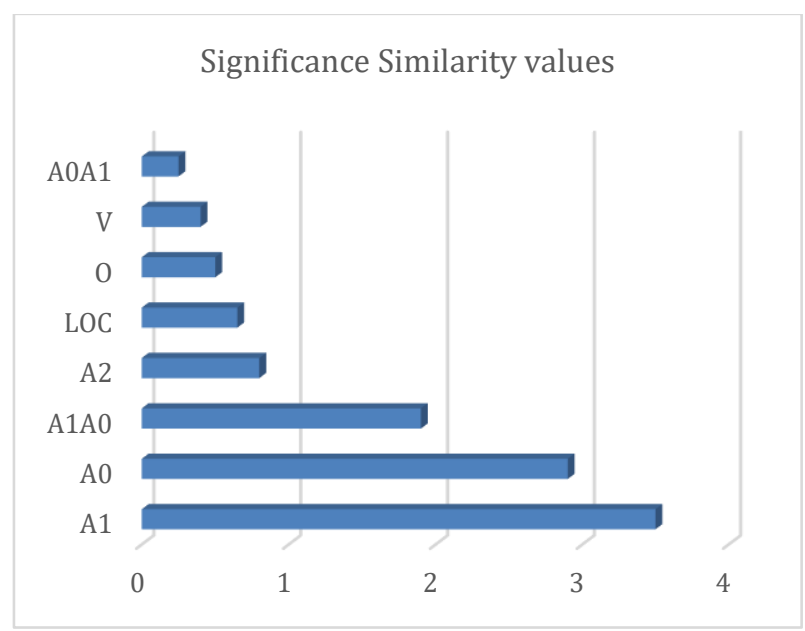

Fig. 5: Significant roles using SVM technique

Fig. 5 shows the imperative roles that were chosen by SVM algorithm. The segment shows either factor significance, which demonstrates the relative importance of every role in assessing the SVM display.

The X-pivot demonstrates the relative significance estimations of the chose roles. In light of the SVM algorithm, if the relative estimation of the roles more prominent than 0 , then the roles will be named significant, generally the roles will be delegated insignificant. Then again, Y-pivot demonstrates that they chose critical roles between all the data roles. The chose roles are $(\mathrm{A} 0, \mathrm{~A} 1, \mathrm{~A} 2$, A1A0, A0A1, O, Loc, and V) and whatever remains of the roles (TMP, MNR, NEG, DIS, ADV, A3, PNC, A4, MOD, EXT and DIR,) were not chosen as essential roles. Table 5 demonstrates that determination of roles by utilizing technique gives a decent outcome for the similarity location when contrasted with results got from the examination of all roles without segregation.

Table 5: Assessment results after the significant roles

\begin{tabular}{|c|c|c|c|}
\hline Cluster No & Recall & Precision & F-Measure \\
\hline Cluster1 & 0.94 & 0.81 & 0.870171 \\
\hline Cluster2 & 0.92 & 0.86 & 0.888989 \\
\hline Cluster3 & 0.85 & 0.86 & 0.854971 \\
\hline Cluster4 & 0.94 & 0.89 & 0.914317 \\
\hline Cluster5 & 0.93 & 0.92 & 0.924973 \\
\hline
\end{tabular}

Some measurable essentialness tests were implemented (t-tests) and they indicated enhancements accomplished by the suggested technique.

Table 6 shows the quantity of cases, standard deviations, mean values, standard error and significant values for the sets of the previous factors, then after the fact improvement of the Recall, Precision1, and F-measure before and after improvement using SRL-SVM strategy for contrasted and the roles samples t-test methodology. The t-test strategy thinks about the methods for two factors that speak to a similar group at various circumstances. The mean estimations of the two factors of ((Recall 1 and 2); (Precision 1 and 2); and (F-measure 1 and 2)) before and after roles significant selection are shown in the t-test Statistics. Low hugeness esteem for the t-test (commonly under 0.05) demonstrates that there is a huge distinction between the two factors. The obtained results are; Recall (0.005), Precision (0.005) and Fmeasure (0.002), this condition was underscored and its outperformance in assessment measures, which implies the suggested strategy acquired huge outcomes in the Recall, Precision, and F-measure. The certainty interim for the mean distinction does not contain zero; this likewise shows the distinction is huge. Likewise, the hugeness esteem is low in the Recall, Precision, and F-measure values and the certainty interim for the mean contrast does not contain zero. Subsequently, presume is a noteworthy distinction between results prior and then afterward enhancement.

Fig. 6 exhibits the examination between SRL-SVM techniques with alternate strategies copyright infringement identification strategy. 
Table 6: Statistical Significance testing using t-test

\begin{tabular}{|c|c|c|c|c|c|c|}
\hline \multirow{3}{*}{ Performance Measure } & \multicolumn{5}{|c|}{ Differences between Recalls, Precisions and $F$-measures before and after the improvement } & \multirow{3}{*}{ Sig. (2-tailed) } \\
\hline & \multirow[t]{2}{*}{ Mean } & \multirow[t]{2}{*}{ Std. Deviation } & \multirow[t]{2}{*}{ Std. Error Mean } & \multicolumn{2}{|c|}{$\begin{array}{l}\text { 95\% Confidence Interval of the } \\
\text { Difference }\end{array}$} & \\
\hline & & & & Lower & Upper & \\
\hline Recall1- Recall2 & -.0906 & .0370 & .0165 & -.1366 & -.0445 & 0.005 \\
\hline Precision1-Precision2 & -.1934 & .0762 & .0341 & -.2880 & -.0987 & 0.005 \\
\hline$F$-measure1-F-measure2 & -.148855 & .0488 & .0218 & -.2095 & -.0881 & 0.002 \\
\hline
\end{tabular}

Comparison between SRL-SVM and other Plagiarism Methods

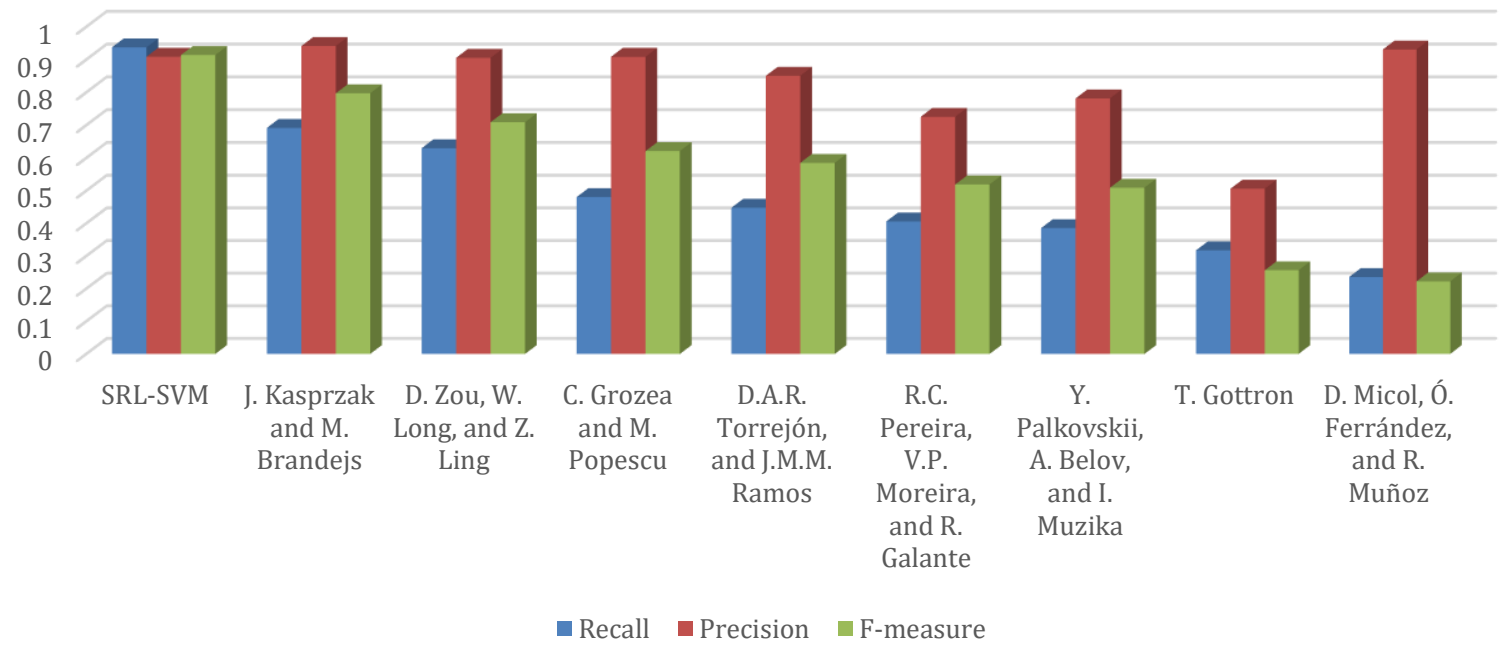

Fig. 6: The evaluations comparison between the suggested technique and the other techniques

The introduced strategy accomplished great outcomes as far as Recall and F-measure. The proposed technique accomplished a typical execution in exactness calculate, yet at the same time superior to anything a portion of alternate strategies to the future goal is to enhance the precision measure to be more precise.

Several of the plagiarism detection systems obtained $0\left(\mathrm{n}^{2}\right)$ class based on JPlag (Prechelt et al., 2002; Mozgovoy et al., 2005). Where $n$ is the an input size (number of documents) of the dataset, and $\mathrm{f}(\mathrm{n})$ is the comparison time between one pair of documents of size $n$. A sample of comparison between the proposed method and other plagiarism detection method in term of the time complexity shown in Table 7.

Table 7: The time complexity comparisons

\begin{tabular}{cc}
\hline Method & $\begin{array}{c}\text { Time } \\
\text { Efficiency }\end{array}$ \\
\hline Graph-based Method (Osman et al., 2011; Osman \\
et al., 2010) & $\mathrm{O}(\mathrm{V}+\mathrm{E})$ \\
$\begin{array}{c}\text { Fuzzy Semantic-based String Similarity } \\
\text { (Alzahrani and Salim, 2010) }\end{array}$ & $\mathrm{O}\left(\mathrm{n}^{2}\right)$ \\
LCS (Elhadi and Al-Tobi, 2008) & $\mathrm{O}\left(\mathrm{n}^{2}\right)$ \\
Semantic-based similarity (Kent and Salim, 2010) & $\mathrm{O}\left(\mathrm{n}^{2}\right)$ \\
SRL-SVM & $\mathrm{O}\left(\mathrm{n}^{2}\right)$ \\
\hline
\end{tabular}

Table 7 shows the time efficiency comparison between SRL-SVM with graph-based method, fuzzy semantic string similarity, and LCS, and semanticbased similarity, detection. The time efficiency of the suggested technique was additionally ascertained and it is has a place in the $\mathrm{O}\left(\mathrm{n}^{2}\right)$ Class.

\section{Discussion}

In this paper, a semantic copyright infringement identification scheme in view of an SRL and SVM strategy was proposed and talked about. The proposed strategy dissected and looked at the text in light of semantic distributions for each word inside a text. SRL offered huge focal points when it came to producing roles for each sentence semantically. They used to catch the semantic likeness between the sentences. Just the most essential roles as chosen by SVM strategy were utilized as a part of the similitude estimation handle. Picking every role created by the SVM algorithm keeping in mind the end goal to choose critical roles was another component of the SVM as opposed to arrangement errand. Not all roles in a text will affect the copyright infringement discovery handle and thus, just the most critical roles were chosen by the SVM algorithm and the outcomes have been utilized as a part of the comparability computation process. The outcomes of the test tests against the PAN-PC-10 data collections demonstrated that the general of the proposed technique execution is accomplished better outcomes. The outcomes additionally uncovered that the proposed strategy in view of the SVM technique can spatially enhance SRL plagiarism recognition. The speculation displayed the possibility that the nature of counterfeiting discovery can be enhanced utilizing SVM method. The concentration of the proposed strategy was balanced so that only the most imperative roles got consideration. Thus, the 
execution was improved. T-Tests were performed to look at the upgrades accomplished by the proposed strategy previously, then after the fact critical roles determination. The consequences of the T-Tests found the advantages of the proposed strategy examined in this paper were measurably huge. One of the limitations of the proposed method it cannot detect the cross-language semantic plagiarism spatially when the text translated from language to another with adding grammar rules of the translated language.

\section{Conclusion and future works}

This study inferred that the critical roles were anticipated utilizing the SVM algorithm. The semantic Role Labeling was utilized for the copyright infringement location by extricating sentence roles and looking at the roles. The impacts of these roles were considered, and the roles have been chosen to utilize an SVM algorithm. Later on, an integration of SRL-SVM with translator method will be introduced as advanced strategies to enhance the limitation of the SRL-SVM technique.

\section{Acknowledgment}

This work is supported by King Abdulaziz University. The authors would like to thank the Deanship of Scientific Research Management (DSR) King Abdulaziz University for the support and incentive extended in making this study a success.

\section{References}

Alzahrani S and Salim N (2010). Fuzzy semantic-based string similarity for extrinsic plagiarism detection (Lab report for PAN@ CLEF10). In the $4^{\text {th }}$ International Workshop PAN-10, Padua, Italy.

Alzahrani SM, Salim N, and Abraham A (2012). Understanding plagiarism linguistic patterns, textual features, and detection methods. IEEE Transactions on Systems, Man, and Cybernetics, Part C (Applications and Reviews), 42(2): 133 149.

Buckley C, Salton G, Allan J, and Singhal A (1995). Automatic query expansion using SMART: TREC 3. In: Harman DK (Ed.), The Third Text REtrieval Conference (TREC3): 69-80. National Institute of Standards and Technology Special Publication, Gaithersburg, Maryland, USA.

Burrows S, Potthast M, and Stein B (2013). Paraphrase acquisition via crowdsourcing and machine learning. ACM Transactions on Intelligent Systems and Technology (TIST), 4(3): 1-21.

Chhabra P, Wadhvani R, and Shukla S (2010). Spam filtering using support vector machine. Special Issue IJCCT, 1(2): 161-171.

Elhadi M and Al-Tobi A (2008). Use of text syntactical structures in detection of document duplicates. In the $3^{\text {rd }}$ International Conference on Digital Information Management, IEEE, London, UK: 520-525. https://doi.org/10.1109/ICDIM.2008. 4746719

Elhadi M and Al-Tobi A (2009). Duplicate detection in documents and webpages using improved longest common subsequence and documents syntactical structures. In the Fourth International Conference on Computer Sciences and Convergence Information Technology, IEEE: 679-684. https://doi.org/10.1109/ICCIT.2009.235
Frakes WB and Baeza-Yates R (1992). Information retrieval: data structures and algorithms. Prentice-Hall, Inc. Upper Saddle River, USA

Franco-Salvador M, Rosso P, and Montes-y-Gómez M (2016). A systematic study of knowledge graph analysis for crosslanguage plagiarism detection. Information Processing and Management, 52(4): 550-570.

Ghosh A, Bhaskar P, Pal S, and Bandyopadhyay S (2011). Rule based plagiarism detection using information retrieval. Jadavpur University, Kolkata, India.

Gipp B (2014). Citation-based plagiarism detection. Springer Vieweg Research, Berlin, Germany.

Gruner S and Naven S (2005). Tool support for plagiarism detection in text documents. In the ACM Conference on Applied Computing, ACM, Santa Fe, New Mexico, USA: 776781. https://doi.org/10.1145/1066677.1066854

Jin Q and Ming M (2011). A method to construct self-set for IDS based on negative selection algorithm. In the International Conference on Mechatronic Science, Electric Engineering and Computer, IEEE, Jilin, China: 1051-1053. https://doi.org/ 10.1109/MEC.2011.6025646

Kent C and Salim N (2010). Features based text similarity detection. Journal of Computing, 2(1): 53-57.

Kim H, Kang YK, Kwon PJ, and Kim MH (2005). An application of DICOM architecture for detecting plagiarism in natural language. In the $9^{\text {th }}$ International Conference on Computer Supported Cooperative Work in Design, IEEE, Coventry, UK: 2: 816-819. https://doi.org/10.1109/CSCWD.2005.194290

Koroutchev K and Cebrián M (2006). Detecting translations of the same text and data with common source. Journal of Statistical Mechanics: Theory and Experiment, 2006(10). https://doi.org/10.1088/1742-5468/2006/10/P10009

Lennon M, Pierce DS, Tarry BD, and Willett P (1981). An evaluation of some conation algorithms for information retrieval. Journal of Information Science, 3(4): 177-183.

Màrquez L, Carreras X, Litkowski KC, and Stevenson S (2008). Semantic role labeling: an introduction to the special issue. Computational Linguistics, 34(2): 145-159.

Mikheev A (2000). Document centered approach to text normalization. In the $23^{\text {rd }}$ Annual International ACM SIGIR Conference on Research and Development in Information Retrieval, ACM, Athens, Greece: 136-143. https://doi.org/ $10.1145 / 345508.345564$

Mikut R and Reischl M (2011). Data mining tools. Wiley Interdisciplinary Reviews: Data Mining and Knowledge Discovery, 1(5): 431-443.

Mozgovoy M, Fredriksson K, White D, Joy M, and Sutinen E (2005). Fast plagiarism detection system. In the International Conference on String Processing and Information Retrieval, Springer Berlin Heidelberg, Heidelberg, Germany: 267-270. https://doi.org/10.1007/11575832_30

Noble WS (2006). What is a support vector machine?. Nature Biotechnology, 24(12): 1565-1567.

Osman AH and Salim N (2013). An improved semantic plagiarism detection scheme based on Chi-squared automatic interaction detection. In the International Conference on Computing, Electrical and Electronics Engineering, IEEE, Khartoum, Sudan: 640-647. https://doi.org/10.1109/ICCEEE.2013. 6634015

Osman AH, Salim N, and Binwahlan MS (2010). Plagiarism Detection Using Graph-Based Representation. Journal of Computing, 2(4): 36-41.

Osman AH, Salim N, and Elhadi AAE (2013). A tree-based conceptual matching for plagiarism detection. In the International Conference on Computing, Electrical and Electronics Engineering, IEEE, Khartoum, Sudan: 571-579. https://doi.org/10.1109/ICCEEE.2013.6634003 
Osman AH, Salim N, Binwahlan MS, Alteeb R and Abuobieda A (2012a). An improved plagiarism detection scheme based on semantic role labeling. Applied Soft Computing, 12(5): 14931502.

Osman AH, Salim N, Binwahlan MS, Hentably H, and Ali MA (2011). Conceptual similarity and graph-based method for plagiarism detection. Journal of Theoretical and Applied Information Technology, 32(2): 135-145.

Osman AH, Salim N, Binwahlan MS, Twaha S, Kumar YJ, and Abuobieda A (2012b). Plagiarism detection scheme based on Semantic Role Labeling. In the International Conference on Information Retrieval and Knowledge Management, IEEE, Kuala Lumpur, Malaysia: 30-33. https://doi.org/10.1109/ InfRKM.2012.6204978

Ozgencil N, Mccracken N, and Mehrotra K (2008). A cluster-based classification approach to semantic role labeling. In: Nguyen NT, Borzemski L, Grzech A, and Ali M (eds.), New Frontiers in Applied Artificial Intelligence: 265-275. Springer, Berlin, Germany.

Palkovskii Y, Belov A, and Muzyka I (2011). Using WordNet-based semantic similarity measurement in external plagiarism detection. In the $5^{\text {th }}$ International Workshop on Uncovering Plagiarism, Authorship, and Social Software Misuse. Notebook Papers of CLEF. Available online at: http://clef2011.org/ resources/proceedings/Palkovskii-Clef2011.pdf

Palmieri F, Fiore U, and Castiglione A (2014). A distributed approach to network anomaly detection based on independent component analysis. Concurrency and Computation: Practice and Experience, 26(5): 1113-1129.

Paul M and Jamal S (2015). An improved SRL based plagiarism detection technique using sentence ranking. Procedia Computer Science, 46: 223-230.

Potthast M, Barrón-Cedeño A, Eiselt A, Stein B, and Rosso P (2010a). Overview of the $2^{\text {nd }}$ International Competition on Plagiarism Detection. In the $4^{\text {th }}$ Workshop on Uncovering Plagiarism, Authorship, and Social Software Misuse, Notebook Papers of CLEF. Available online at: https://pdfs.semanticscholar.org/44e2/8a94f857cb5f7702a7 b86455416726df64e9.pdf

Potthast M, Stein B, Barrón-Cedeño A, and Rosso P (2010b). An evaluation framework for plagiarism detection. In the $23^{\text {rd }}$ International Conference on Computational Linguistics: Posters, Association for Computational Linguistics, Beijing, China: 997-1005.

Prechelt L, Malpohl G, and Philippsen M (2002). Finding plagiarisms among a set of programs with JPlag. Journal of Universal Computer Science UCS, 8(11): 1016-1038.

Salcedo-Campos F, Díaz-Verdejo J, and García-Teodoro P (2012). Segmental parameterisation and statistical modelling of $\mathrm{e}$ mail headers for spam detection. Information Sciences, 195: 45-61.

Salim N, Suanmali L, and Binwahlan MS (2010). SRL-GSM: A hybrid approach based on semantic role labeling and general statistic method for text summarization. Journal of Applied Sciences, 10(3): 166-173.

Seaward L and Matwin S (2009). Intrinsic plagiarism detection using complexity analysis. In the $25^{\text {th }}$ Annual Conference of the Spanish Society for Natural Language Processing (SEPLN'09), San Sebastian, Spain: 56-61. Available online at: http://ceur-ws.org/Vol-502/pan09-proceedings.pdf\#page $=64$

Sharma A, Pujari AK, and Paliwal KK (2007). Intrusion detection using text processing techniques with a kernel based similarity measure. Computers and Security, 26(7): 488-495.

Shehata S, Karray F, and Kamel MS (2010). An efficient model for enhancing text categorization using sentence semantics. Computational Intelligence, 26(3): 215-231.

Stamatatos E (2009). A survey of modern authorship attribution methods. Journal of the American Society for information Science and Technology, 60(3): 538-556.

Stamatatos E (2009). Intrinsic plagiarism detection using character n-gram profiles. In the Annual Conference of the Spanish Society for Natural Language Processing (SEPLN'09), Donostia, Spain: 38-46. Available online at: http://ceurws.org/Vol-502/paper8.pdf

Stein B, Lipka N, and Prettenhofer P (2011). Intrinsic plagiarism analysis. Language Resources and Evaluation, 45(1): 63-82.

Suárez P, González JC, and Román JV (2010). A Plagiarism Detector for Intrinsic, External and Internet Plagiarism. In Notebook Papers of CLEF 2010 LABs and Workshops, Padua, Italy.

Temitayo F, Stephen O, and Abimbola A (2012). Hybrid GA-SVM for efficient feature selection in e-mail classification. Computer Engineering and Intelligent Systems, 3(3): 17-28.

Tomasic A and Garcia-Molina H (1993). Query processing and inverted indices in shared: nothing text document information retrieval systems. The VLDB Journal-The International Journal on Very Large Data Bases, 2(3): 243-276.

van Rijsbergen CJ (1979). A new theoretical framework for information retrieval. In the $9^{\text {th }}$ annual international ACM SIGIR Conference on Research and development in information retrieval, ACM, Palazzo dei Congressi, Pisa, Italy: 194-200. https://doi.org/10.1145/253168.253208

Wang L (2005). Support vector machines: theory and applications. Springer Science and Business Media, Berlin, Germany.

Youn S and McLeod D (2007). A comparative study for email classification. In: Elleithy K (Ed.), Advances and innovations in systems, computing sciences and software engineering: 387 391. Springer, Amsterdam, Netherlands.

Zou D, Long WJ, and Ling Z (2010). A cluster-based plagiarism detection method. In the Notebook Papers of CLEF 2010 LABs and Workshops, Padua, Italy. Available online at: http://www.uni-weimar.de/medien/webis/events/pan10/pan10-papers-final/pan10-plagiarism-detection/du10notebook.pdf 